Ophthalmologe 2022 $1119: 1051-1053$ https://doi.org/10.1007/s00347-021-01542-x Eingegangen: 11. Oktober 2021 Überarbeitet: 10. November 2021 Angenommen: 11. November 2021 Online publiziert: 4. Dezember 2021 (c) Der/die Autor(en) 2021

\section{Zysten des Irispigmentepithels}

Reinhard Told (D) · Adrian Reumueller · Martin Zehetmayer · Roman Dunavölgyi

Universitätsklinik für Augenheilkunde und Optometrie, Medizinische Universität Wien, Wien, Österreich

\section{Kasuistik}

Ein 56-jähriger, männlicher Patient wurde an die Universitätsklinik für Augenheilkunde und Optometrie, Medizinische Universität Wien mit Verdacht auf Ziliarkörpermelanom zugewiesen, um einen pigmentierten Iristumor des rechten Auges weiter abzuklären.

\section{Anamnese}

Bei einer Routineuntersuchung durch den niedergelassenen Augenfacharzt wurde eine pigmentierte Irisläsion festgestellt. Der Patient war subjektiv beschwerdefrei. Es lagen keine systemischen Erkrankungen oder medikamentösen Therapien vor. In der Augenanamnese fanden sich eine komplikationslose Operation des grauen Stars ca. 6 Monate sowie eine Nd:YAGKapsulotomie ca. 2 Monate zuvor.

\section{Befund}

Der bestkorrigierte Snellen-Visus war an beiden Augen 1,0. Bei der Spaltlampenun-

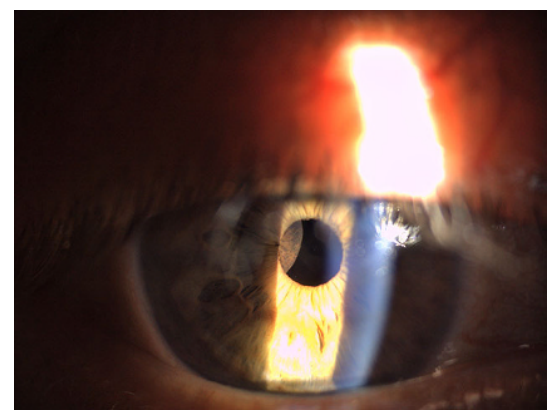

Abb. $1 \Delta$ Die Spaltlampenfotografie des rechten Auges zeigt eine braune, rundliche Masse, welche sich von retropupillar vorwölbt tersuchung vor dem Erweitern der rechten Pupille zeigte sich eine gut umschriebene, fusiforme braune Masse, welche sich, ausgehend von der Irisrückfläche, bei etwa $10 \mathrm{Uhr}$ in die Pupille vorwölbte. Es zeigten sich ein homogenes Erscheinungsbild ohne intrinsische Gefäße, Feeder- oder Sentinelgefäße, kein Ektropium der Iris und keine Korektopie (- Abb. 1). Das Weitstellen der Pupille führte zu einem ausgeprägten Erscheinungsbild der fusiformen, pigmentierten Läsion zwischen 7 und 11 Uhr (- Abb. 2). Eine Ultraschallbiomikroskopie(UBM)-Untersuchung vor der Pupillenerweiterung zeigte eine große elongierte Zyste von ca. $4 \mathrm{~mm}$ Länge und einer Dicke von ca. $1 \mathrm{~mm}$ bei 9 Uhr an der Irisrückfläche (• Abb. 3). Bei 10 Uhr 30 zeigten sich multiple kleinere Zysten im Bereich der Iriswurzel (• Abb. 4). Der transversale UBM-Scan an der Iriswurzel (• Abb. 5) zeigte für Zysten klassische mehrkammerige, schallleere Hohlräume mit hochechogener, dünnwandiger, scharfer Begrenzung. Im Bereich der solitären großen Zyste war der Kammerwinkel verlegt (-Abb. 3). Es fanden sich weder

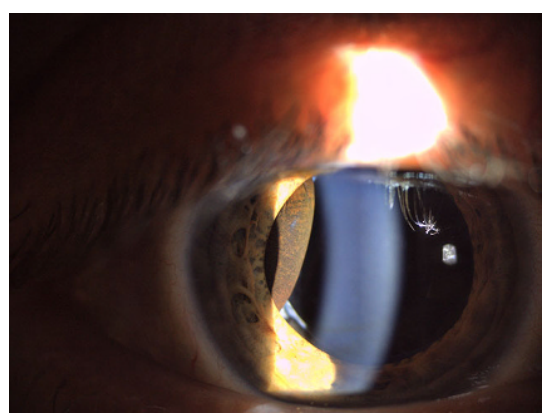

Abb. $2 \triangle$ Das Spaltlampenfoto nach Pupillenweitstellung zeigt eindrücklich die braune Läsion, welche sich im Bereich von 8 bis 11 Uhr präsentiert 


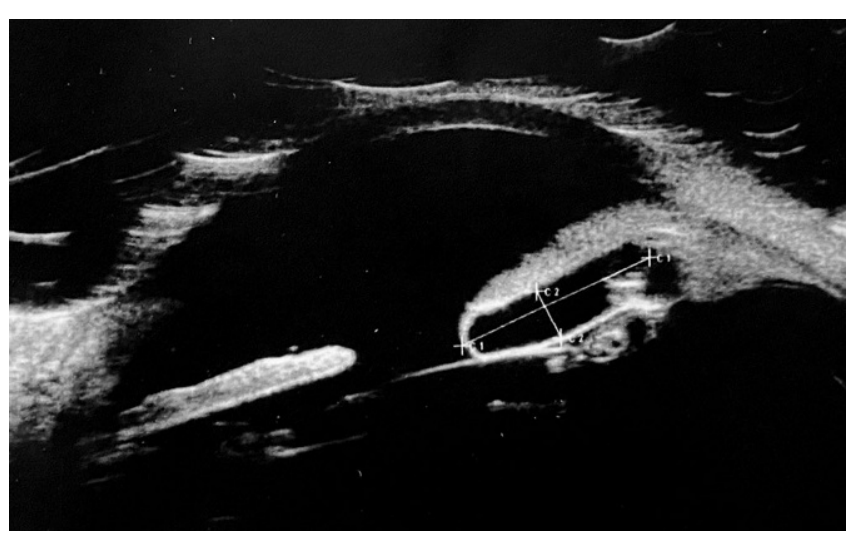

Abb. 3 A Die UBM-Untersuchung zeigt die große, elongierte Zyste von ca. $4 \mathrm{~mm}$ Länge und $1 \mathrm{~mm}$ Höhe. Dahinter zeigt sich die Kunstlinse. In der Zyste sind die Messmarker abgebildet

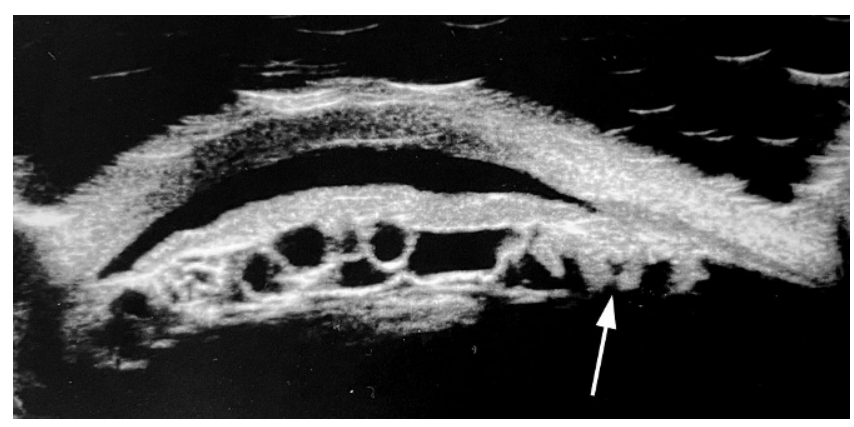

Abb. $5<$ Multiple, kleine Iriszysten im transversalen Schnitt der UBMUntersuchung. Der weiße Pfeil zeigt auf die Ziliarkörperzotten

Anzeichen für eine solide Masse noch für ein malignes Geschehen. Die Fundoskopie in Mydriase war unauffällig.

\section{Diagnose}

Als Diagnose wurden Zysten des Irispigmentepithels gestellt.

\section{Therapie und Verlauf}

Bei subjektiver Beschwerdefreiheit wurde eine periodische Observanz 2-mal jährlich beim Augenfacharzt empfohlen.

\section{Diskussion}

Zysten des Irispigmentepithels können beim untersuchenden Augenfacharzt den Verdacht auf ein solides Geschehen, wie z. B. ein Melanom des Ziliarkörpers [1], erwecken. Die Zysten des Irispigmentepithels präsentieren sich als braune, rundliche oder spindelförmige (fusiforme) Läsion und treten oft erst nach Weitstellen der Pupille in Erscheinung. An der Spaltlampe haben sie eine glatte bis samtige Oberfläche. Typischerweise befinden sich die Zysten an der Irisrückfläche im Bereich des Pigmentepithels und werden in der englischen Literatur anhand ihrer Lage als „midzonal“ oder „peripheral iris pigment epithelial cysts" bezeichnet. Sie besitzen in der OCT- oder UBM-Untersuchung eine hoch reflektive, kugelige bis ellipsoide, begrenzende Wand, der Inhalt jedoch ist von sehr geringer bis keiner Reflektivität. Der Ursprung der Zysten ist nicht abschließend geklärt. Die vermuteten Ursachen von Zysten des Ziliarkörpers reichen von angeborenen Zysten bis zu mechanischen Ursachen im Rahmen der Akkommodation [2]. Dies trifft möglicherweise auch auf Zysten des Irispigmentepithels zu. Zysten des Irispigmentepithels verhalten sich typischerweise ruhig und werden im Verlauf observiert. In seltenen Fällen können die Zysten lageabhängig die optische Achse verlegen oder, wenn sie peripher auftreten, das Irisstroma nach anterior verlagern und folglich den Kammerwinkel verschließen. Differenzialdiagnostisch müssen Adenome des Irispigmentepithels [3] sowie solide Prozesse der Iris wie ein Melanom berücksichtigt werden - Letzteres auch vor dem Hintergrund, dass
Irismelanome in sehr seltenen Fällen ein zystisches Erscheinungsbild aufweisen können [4]. Hier können Spaltlampenund Ultraschalluntersuchung wichtige Hinweise geben, da im angeführten Fall [4] episklerale Sentinelgefäße beschrieben wurden und eine solide basale Komponente vorhanden war. Adenome sowie Zysten des Irispigmentepithels weisen in der Regel keine erweiterten episkleralen Gefäße auf.

Therapeutisch kann eine Feinnadelpunktion mit Zystendeflation angewendet werden [5]. Des Weiteren kann eine Neodymium-YAG-Lasertherapie der Zyste erfolgen, um diese zu eröffnen [6].

Das klinische Erscheinungsbild in Zusammenschau mit Methoden zur Darstellung des vorderen Augenabschnittes wie UBM oder optische Kohärenztomographie (OCT) des vorderen Augenabschnittes kann dem Augenfacharzt bei der Unterscheidung zwischen Iriszysten und soliden Prozessen helfen, um ein vermutetes Melanom rasch ausschließen zu können.

\section{Fazit für die Praxis}

Das klinische Erscheinungsbild in Zusammenschau mit Methoden zur Darstellung des vorderen Augenabschnittes wie UBM oder OCT kann dem Augenfacharzt bei der Unterscheidung zwischen Iriszysten und soliden Prozessen helfen, um ein vermutetes Melanom rasch ausschließen zu können. 


\section{Fachnachrichten}

\section{Korrespondenzadresse}

\section{PD Dr. Reinhard Told, PhD}

Universitätsklinik für Augenheilkunde und Optometrie, Medizinische Universität Wien Währinger Gürtel 18-20, 1090 Wien, Österreich reinhard.told@meduniwien.ac.at

Funding. Open access funding provided by Medical University of Vienna.

\section{Einhaltung ethischer Richtlinien}

Interessenkonflikt. R. Told, A. Reumueller, M. Zehetmayer und R. Dunavölgyi geben an, dass kein Interessenkonflikt besteht.

Für diesen Beitrag wurden von den Autoren keine Studien an Menschen oder Tieren durchgeführt. Für die aufgeführten Studien gelten die jeweils dort angegebenen ethischen Richtlinien. Für Bildmaterial oder anderweitige Angaben innerhalb des Manuskripts, über die Patienten zu identifizieren sind, liegt von ihnen und/oder ihren gesetzlichen Vertretern eine schriftliche Einwilligung vor.

Open Access. Dieser Artikel wird unter der Creative Commons Namensnennung 4.0 International Lizenz veröffentlicht, welche die Nutzung, Vervielfältigung, Bearbeitung, Verbreitung und Wiedergabe in jeglichem Medium und Format erlaubt, sofern Sie den/die ursprünglichen Autor(en) und die Quelle ordnungsgemäß nennen, einen Link zur Creative Commons Lizenz beifügen und angeben, ob Änderungen vorgenommen wurden.

Die in diesem Artikel enthaltenen Bilder und sonstiges Drittmaterial unterliegen ebenfalls der genannten Creative Commons Lizenz, sofern sich aus der Abbildungslegende nichts anderes ergibt. Sofern das betreffende Material nicht unter der genannten Creative Commons Lizenz steht und die betreffende Handlung nicht nach gesetzlichen Vorschriften erlaubt ist, ist für die oben aufgeführten Weiterverwendungen des Materials die Einwilligung des jeweiligen Rechteinhabers einzuholen.

Weitere Details zur Lizenz entnehmen Sie bitte der Lizenzinformation auf http://creativecommons.org/ licenses/by/4.0/deed.de.

\section{Literatur}

1. Costache M, Patrascu OM, Adrian D, Costache D, Sajin M, Ungureanu E, Simionescu O (2013) Ciliary body melanoma - a particularly rare type of ocular tumor. Case report and general considerations. Maedica (Bucur) 8(4):360-364

2. Rohrbach JM, Steuhl KP, Thiel HJ (1991) Cysts and Fuchs' endothelial adenomas of the pars plicata corporis ciliaris-degeneration products as an expression of various ciliary body functions? Klin Monbl Augenheilkd 198(3):195-200. https://doi. org/10.1055/s-2008-1045951

3. Shields JA, Shields CL, Mercado G, Gunduz K, Eagle RC Jr (1999) Adenoma of the iris pigment epithelium: a report of 20 cases: the 1998 Pan-Amer- ican Lecture. Arch Ophthalmol 117(6):736-741. https://doi.org/10.1001/archopht.117.6.736

4. Zhang J, Demirci H, Shields CL, Leon JA, Shields JA Eagle RC Jr (2005) Cavitary melanoma of ciliary body simulating a cyst. Arch Ophthalmol 123(4):569-571. https://doi.org/10.1001/ archopht.123.4.569

5. Shields JA, Fallon SJ, Shields CN, Lally SE, Shields CL (2018) Advanced midzonal iris pigment epithelial cyst managed by aspiration deflation. Ocul Oncol Pathol 4(3):182-185. https://doi.org/10.1159/ 000481405

6. Schrems W, Tomlinson GP, Belcher CD 3rd (1986) Neodymium-YAG laser therapy for iris cysts. Arch Ophthalmol 104(8):1130-1134. https://doi.org/ 10.1001/archopht.1986.01050200036033
DOG-Kongress 2022

- Abstracts online unter www.DieOphthalmologie.de

Vom 29.09. - 02.10.2022 fand der DOG Kongress 2022 in Berlin statt.

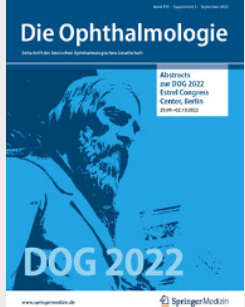

\section{Abstracts} online

Die Abstracts zum Kongress sind auch dieses Jahr als Online-Supplement der Zeitschrift Die Oph-

thalmologie erschienen. Das Supplement informiert detailliert über die Inhalte des Kongresses und stellt gleichzeitig für alle Autorinnen und Autoren die Zitierfähigkeit ihrer Abstracts sicher.

Unter folgendem Link können Sie die Abstracts nachlesen: https://link.springer.com/article/ 10.1007/s00347-022-01723-2

Oder scannen Sie diesen QR-Code ein:

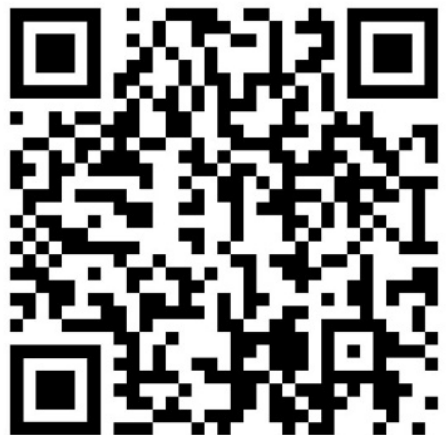

Ebenfalls finden Sie den Abstractband auf der Zeitschriftenhomepage www.DieOphthalmologie.de über den Link „1997-2022". Wählen Sie dort „Ausgabe Sonderheft 3/2022", um zu den Abstracts zu gelangen. 